\title{
Data processing in luminescence dating analysis: an exemplary workflow using the $\mathbf{R}$ package 'Luminescence'
}

\author{
Margret C. Fuchs ${ }^{\mathrm{a}, \mathrm{b}, *}$, Sebastian Kreutzer ${ }^{\mathrm{c}}$, Christoph Burow $^{\mathrm{d}}$, Michael Dietze $^{\mathrm{e}}$, Manfred Fischer $^{\mathrm{f}}$, Christoph Schmidt $^{\mathrm{f}}$, \\ Markus Fuchs ${ }^{\mathrm{C}}$ \\ ${ }^{a}$ Department of Periglacial Research, Alfred-Wegener-Institute for Polar and Marine Research, 14473 Potsdam, Germany \\ ${ }^{b}$ Luminescence Laboratory, Institute of Applied Physics, TU Bergakademie Freiberg, 09596 Freiberg, Germany \\ ${ }^{c}$ Department of Geography, Justus-Liebig-University Giessen, 35390 Giessen, Germany \\ ${ }^{d}$ Institute for Geography, University of Cologne, 50923 Cologne, Germany \\ e Section 5.1 Geomorphology, GFZ German Research Centre for Geosciences, 14473 Potsdam, Germany \\ ${ }^{f}$ Geographical Institute, Geomorphology, University of Bayreuth, 95440 Bayreuth, Germany
}

\begin{abstract}
The first version of the $\mathbf{R}$ package 'Luminescence' was released and published in 2012. Since then, the package has been continuously improved - by implementing further measurement protocols, adding age models, and extending functions. In geoscientific applications, luminescence dating requires a series of data processing procedures. A comprehensive and replicate analysis of luminescence data using the $\mathbf{R}$ package 'Luminescence', therefore, suggests combining selected functions. With this contribution, we provide a practical example of a workflow from reading measurement data to age modelling. The exemplary data processing routine is applied to an OSL data set of a fluvial sediment sample from the Pamir Mountains.
\end{abstract}

Keywords: CRAN, data processing, OSL, Pamir, R templates

\section{Introduction}

The R package 'Luminescence' (Kreutzer et al., 2012) provides a bundle of functions for the analysis of luminescence signals and related data. The feedback from package users encouraged the developer team to revise, upgrade and add functions, and to release supportive material such as a practical guide for its use (Dietze et al., 2013) and various tutorials, available at the $\mathbf{R}$ Luminescence website (http://www.r-luminescence.de). The current package (version 0.3.3) equips the user with a flexible tool for various data processing steps, such as reading measurement data, fitting growth curves, calculating equivalent doses, inferring dose distribution statistics and applying age models. The open source code guarantees full access to all calculations. One may adapt functions according to individual requirements, taking advantage of the $\mathbf{R}$ programming environment ( $\mathrm{R}$ Core Team, 2014) and any packages available through the Comprehensive $\mathrm{R}$ Archive Network (CRAN, http://cran.r-project.org/).

In luminescence dating, a convenient workflow demands a series of data processing steps to be reproduced for several samples. This suggests the use of $\mathbf{R}$ code templates that define how data are passed through selected functions and how the output is processed. Nevertheless, the application of luminescence methods to a broad range of

\footnotetext{
* corresponding author

Email address: margret.fuchs@awi.de (Margret C. Fuchs)
}

sediments affects various methodological aspects. Consequently, the selection of adequate analytical steps requires adjustment and may be far beyond routine procedures.

Here, we can neither discuss the full range of data processing needs nor provide a standard decision procedure for luminescence data analysis. We rather introduce one example of how the $\mathbf{R}$ package 'Luminescence' can be used. For illustration, we apply selected functions to an optically stimulated luminescence (OSL) data set of fluvial sediments from the Panj river network in Pamir. We focus on a typical workflow from loading the measurement data into the $\mathbf{R}$ environment to statistical age modelling without any age interpretation. The described workflow (Fig. 1) introduces one of many possible routines to encourage the wider geosciences community to use, adapt or combine the available functions and to contribute to the $\mathbf{R}$ package development.

$\mathbf{R}$ code snippets are typed in monospaced letters throughout the manuscript. For data processing, the $\mathbf{R}$ user interface RStudio (http://www.rstudio.com) is used. The described workflow may require adaption for previous $(<0.3 .3)$ package versions. Details on sample data processing are given in three $\mathbf{R}$ code templates (supplementary data).

\section{Sample material and OSL measurement}

A practical example to illustrate one possible workflow for OSL dating analyses is a data set derived from a fluvial sediment sample (TA110817N9-10) from the Panj 
R.Luminescence

Example for data processing

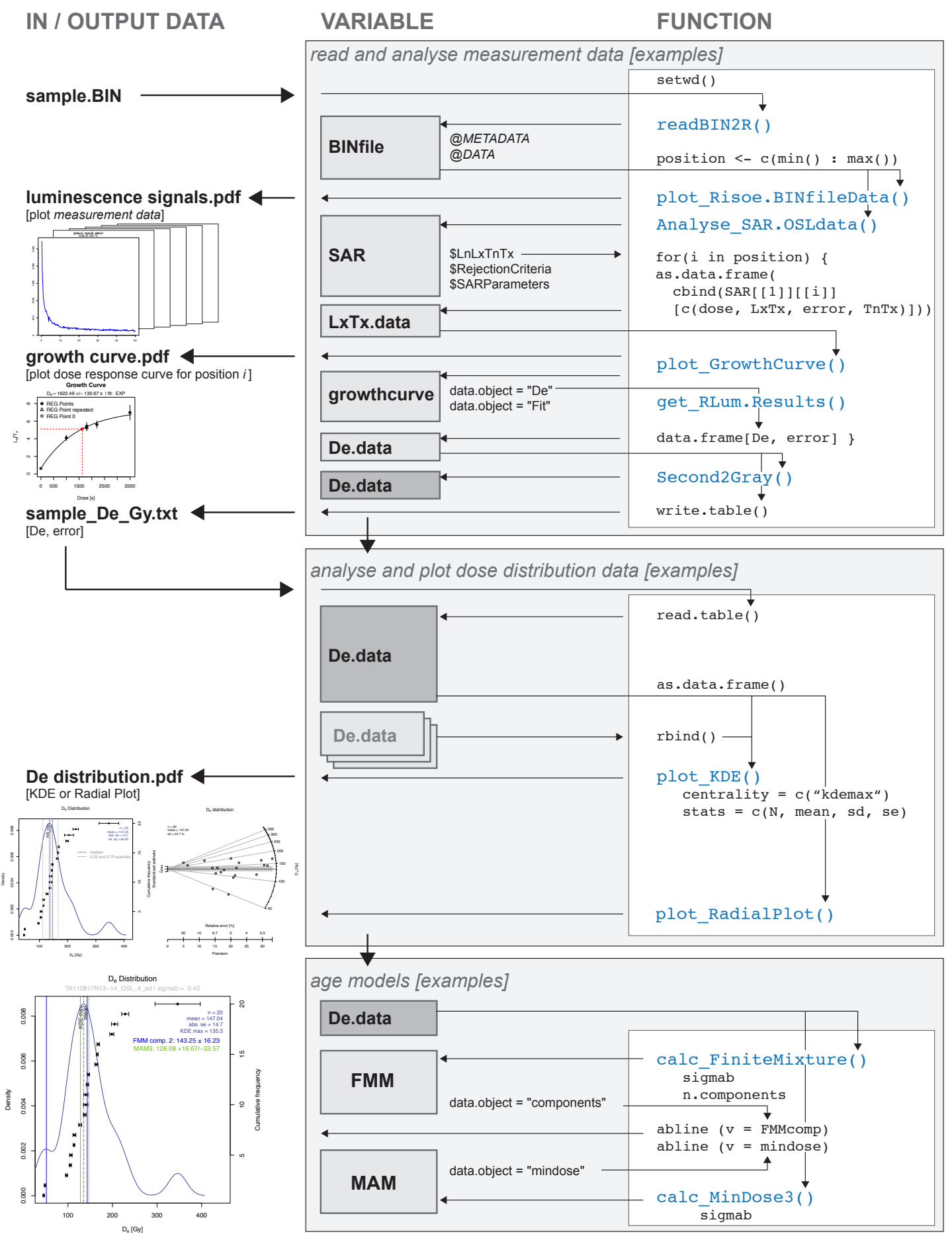

detailed documentation: http://www.r-luminescence.de

Figure 1: Flow chart of exemplary data processing in luminescence dating analysis. Exemplary variable and data names are chosen for straightforward comprehension of the content. The flow chart comprises three major steps (light grey boxes): data input and analyses of measured signal, dose distribution analyses, age modelling. Arrows indicate the flow of variables as modified by R-functions (within/between light grey boxes) or data input and output options (in/out of light grey boxes). $\mathbf{R}$ code snippets are typed in monospaced letters) 
River, situated in the Pamir mountains. The regional geology, climate and the geomorphometry of the drainage system is described in Fuchs et al. (2013). The site is part of a complex terrace system along the Murgab River at the eastern Pamir Plateau (N 38.155012 ${ }^{\circ}$, E $73.968168^{\circ}$, 3,599 ma.s.l.). The sampled sediment consists of wellsorted, layered sand $23 \mathrm{~m}$ below the terrace surface. The measurements to determine the equivalent dose $\left(D_{e}\right)$ for OSL dating were conducted on the coarse-grained quartz fraction $(90-160 \mu \mathrm{m})$, using a single aliquot regenerative dose (SAR) protocol after Murray and Wintle (2000). Based on multiple grain SAR measurements, 20 medium size (4 mm) aliquots were measured. The SAR cycles were first applied to measure the natural signal, followed by six regeneration cycles ( $\mathrm{R} 1$ to $\mathrm{R} 6$ ), including one to measure the recuperation (R5) and one to measure the recycling ratio (R6). Finally, an infrared stimulation cycle was performed using the same dose as for R1. Alongside the 20 aliquots measured for $D_{e}$ determination, four additional aliquots (position 21-24) were measured to record signals from a dose recovery test (Murray and Wintle, 2003). All measurements were conducted using a standard Ris $\varnothing \mathrm{DA}-$ $20 \mathrm{TL} / \mathrm{OSL}$ reader $\left({ }^{90} \mathrm{Sr} /{ }^{90} \mathrm{Y}\right.$ beta source $\sim 5.6 \mathrm{~Gy} / \mathrm{min}$, CW-OSL, blue LEDs at $470 \mathrm{~nm}$ and $90 \%$ optical power, 7.5 mm U340 Hoya filter, Bøtter-Jensen et al., 2000; Thomsen et al., 2006), which stores the measurement data in the .BIN file (version 03) format.

\section{Data processing steps}

The $\mathbf{R}$ package 'Luminescence' offers various functions to process the measurement data, such as any equivalent dose data stored in a .BIN or . BINX file (see supplementary data for a complete list of available functions). The recommended workflow includes three main analytical steps: (1) reading and analysing the measurement data, (2) analysing and plotting the dose distribution data, and (3) applying the age models. Figure 1 illustrates the workflow, including how the data can be handled with selected analytical functions, how variables can be used, and which plots can be generated.

\subsection{Read and analyse measurement data}

In order to analyse the OSL signal properties of the Pamir sample, the measurement data was first loaded into $\mathbf{R}$. The presented example data is stored in a .BIN file. Other file formats such as .BINX are also fully supported. The data was imported by readBIN2R() and stored in a variable, here called BINfile (Fig. 1). The details on the measured sequence are available through metadata by selecting the desired rows and columns of the slot. Luminescence signal data is available through the DATA slot of the BINfile variable.

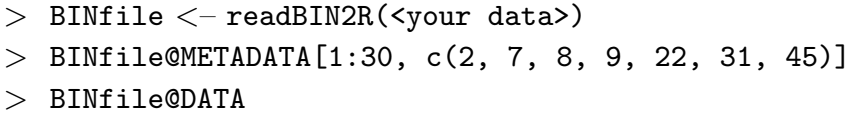

For this data set, aliquot positions 1 to 20 were chosen to investigate the OSL signal properties, while positions 21 to 24 were bleached and dosed before applying the SAR protocol. The function plot_Risoe.BINfileData() plots OSL curve data for each stimulation run of the SAR protocol for all selected positions. The output can be returned to the screen or saved to the working directory using $\mathbf{R}$ internal functions such as pdf().

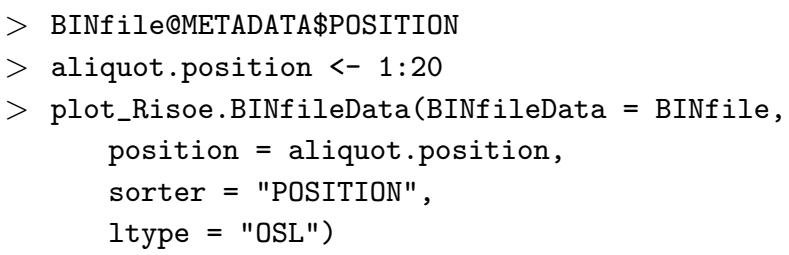

Background and sensitivity corrected initial signals of each OSL curve were calculated and grouped according to position by Analyse_SAR.OSLdata(). The integrals for the initial signal and for the background were set to $1-5$ (1s) and 200-250 (10 s), respectively. Given values represent examples and may be modified due to the individual requirements of analysed data. A variable named $S A R$ was introduced that contains the results of integral calculations for each regeneration cycle $(\mathrm{R} 1-\mathrm{R} 6)$ in the component \$LnLxTnTx (Fig. 1). Additionally, the function returns recycling ratio estimates (R6/R2), recuperation values (R5/ natural signal) in \$RejectionCriteria, and SAR parameters.

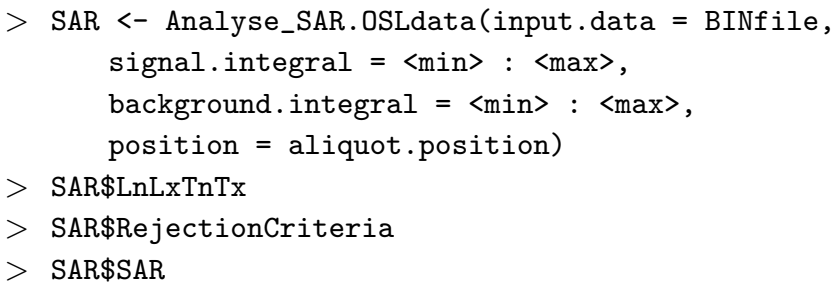

The dose response curve and the $D_{e}$ of the natural signal were determined by fitting exponential curves to the SAR signals ( $L_{x} / T_{x}$ values) of each aliquot. This was performed by the function plot_GrowthCurve(), which requires a data frame of three columns containing regeneration doses, corrected OSL signals, and signal errors. Therefore, the respective data of the selected aliquot position $i$ was passed from SAR $\$ L n L x T n T x$ to a new data frame, here named LxTx.data.

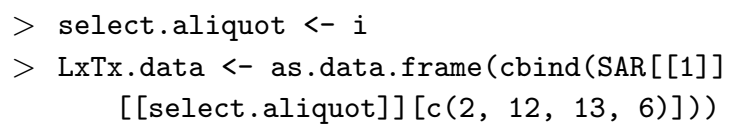

The variable LxTx.data was then used to run the plot_GrowthCurve() function. The output.plot parameter enables printing of results, for example as a .pdf file. Results from the plot_GrowthCurve() function were returned as an "S4 object", here named growthcurve (Fig. 1). Estimated $D_{e}$ and curve fitting parameters were addressed 
through the data slot using the function get_RLum.Results() and selecting, for example, the data object "De". Results may be stored in an empty data.frame() of a certain number of columns and filled with missing values (NA).

$>\operatorname{pdf}()$

$>$ growthcurve <- plot_GrowthCurve (sample $=$ LxTx.data, output.plot $=$ TRUE)

$>\operatorname{dev} . \operatorname{off}()$

$>$ get_RLum.Results (growth.curve, data.object $=$ "De")

$>$ De.data <- data.frame ( De = NA, De.error $=N A)$

De <- get_RLum.Results (growth.curve, data. object $=$ "De")

$>\operatorname{De} \cdot \operatorname{data}[, 1]<-\operatorname{De}[1]$

$>\operatorname{De} \cdot \operatorname{data}[, 2]<-\operatorname{De}[2]$

A two column data frame of equivalent doses and related errors is required for further dose distribution statistics and age models of the $\mathbf{R}$ package 'Luminescence'. An effective way to analyse all selected aliquot positions from measured signal to $D_{e}$ estimate is provided by the loop for () as illustrated in Fig. 1. The loop repeats signal extraction from individual SAR cycle measurements including growth curve estimation for the aliquot positions 1 to 20 , and passes all $D_{e}$ and respective error to a data frame termed De.data (for a detailed example see also the $\mathbf{R}$ template 1 in the supplementary material).

The reliability of the $D_{e}$ calculation can be assessed using, for example, the recycling ratio and recuperation values returned by Analyse_SAR.OSLdata(). Respective data was stored in the slot Rejection.Criteria of the variable $S A R$. Recycling ratio and recuperation of $10 \%$ and $5 \%$, respectively, were included to illustrate $D_{e}$ selection for the Pamir sample. Template 1 (supplementary material) includes an example of how to implement rejection criteria and change thresholds as needed. The data in the variable $S A R$ or growthcurve may be adopted to derive further rejection criteria (e.g., signal to background ratio, $D_{e}$ error) depending on the user's research focus or requirements.

The De.data values of the Pamir sample were given in seconds as irradiation times were given in seconds. To convert the data to the unit Gray (Gy) the package provides the function Second2Gray(). It requires the $D_{e}$ data and the dose rate of the $\beta$-source along with its error. In case the dose rate of the $\beta$-source at the date of measurement is unknown, the $\mathbf{R}$ template 1 (supplementary material) shows an example of the calculation based on the last $\beta$ source calibration and the date of measurement. The variable De.data can be saved as a .txt file for further usage.

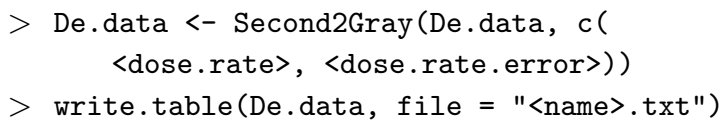

\subsection{Analyse and plot dose distribution data}

The fluvial origin of the Pamir sample demands for statistical analysis of replicated equivalent dose measurements to determine the degree of bleaching and the most likely paleodose (e.g., Galbraith et al., 1999; Bailey and Arnold, 2006; Duller, 2008). Basically, the $\mathbf{R}$ package 'Luminescence' offers three functions for dose distribution visualisation: plot_KDE(), plot_RadialPlot() and plot_Histogram(). Any two-column data can be used as input data: the De.data from previous analyses or any other data frame loaded into the $\mathbf{R}$ environment. The input data may require several preparation steps (see $\mathbf{R}$ template 2 in the supplementary material). This includes selecting the correct data columns with the equivalent doses and errors, removing missing values for aliquot positions which yielded no equivalent dose, and sorting values in ascending order. Although not essential for the plot functions, it is convenient to sort the data for later use with age models (see below and $\mathbf{R}$ template 3 in the supplementary material). Finally, the variable De.data is converted to a data frame.

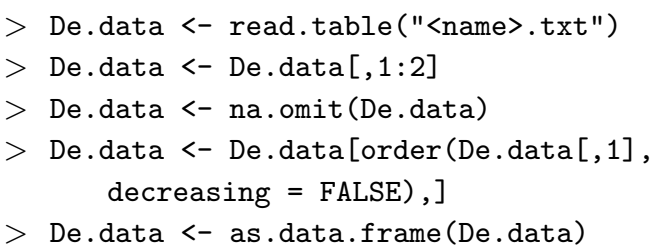

The dose distribution of the Pamir sample was plotted by plot_KDE(). The function illustrates the variability of $D_{e}$ by kernel density estimates (KDE). Basic statistic measures were added to the plot by the parameters centrality for vertical lines indicating the position of central measures within the distribution, and stats for numerical output of descriptive statistics (Fig. 1). The plot output is again saved as a separate .pdf file (see $\mathbf{R}$ template 2 in the supplementary material).

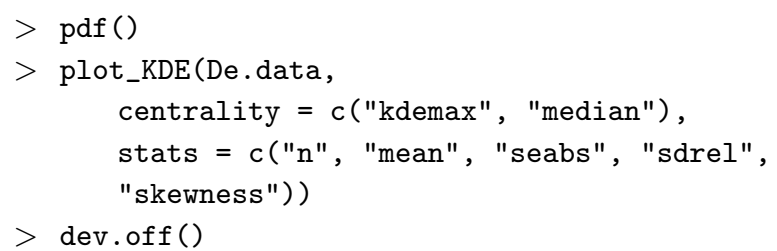

The Pamir example data showed a positively skewed distribution (skewness of 1.13) with the mean higher than the median and the maximum density of $D_{e}$ values. The high standard deviation of $\sim 47 \%$ supports the indication of differential bleaching (e.g., Bailey and Arnold, 2006; Arnold et al., 2007). Consequently, the paleodose calculation is based on further age modelling to extract the well-bleached portion of all $D_{e}$. 


\subsection{Age models}

In the case of abnormal dose distributions, the paleodose calculation based on the arithmetic mean is not appropriate. This leads to an over- or underestimation of the true burial dose, and to miscalculating the luminescence age (e.g., Olley et al., 1998; Murray and Olley, 2002; Rodnight et al., 2006; Bailey and Arnold, 2006; Arnold et al., 2007). For such dose distributions, several age models (e.g., Galbraith and Green, 1990; Galbraith et al., 1999; Fuchs and Lang, 2001; Lepper and McKeever, 2002) have been introduced and are now in common use throughout the published literature. Functions for the most common models are available in the $\mathbf{R}$ package 'Luminescence' (complete list of available age models given in supplementary material). The decision on the appropriate age model is essentially dependent on various aspects concerning the sampled sediments and applied measurement procedure (e.g., Arnold and Roberts, 2009; Arnold et al., 2012). It stays with the user to decide which age model to choose from the set of available functions in the $\mathbf{R}$ package 'Luminescence'. Although the human eye is very good in recognizing patterns, package users may also use statistic measures to evaluate dose distributions. One simple way to identify abnormal distributions is, for example, the comparison of the arithmetic mean and median. Additionally, the $\mathbf{R}$ package 'Luminescence' provides a function calc_Statistics() that returns a number of descriptive statistic estimates such as skewness.

The minimum age model (MAM, Galbraith et al., 1999) was applied to the Pamir sample to address the differential bleaching observed in dose distributions. The variable De.data was again used as the input parameter for the functions calc_MinDose3(). Sigmab is essential for the MAM as an estimate of the sample's overdispersion. A wide range of sigmab from 0.01 to 0.4 was found in fluvial material of different geographical regions (Arnold et al., 2007; Arnold and Roberts, 2009). Hence, a value of 0.3 may be chosen, which is well within the reported range. However, choosing an appropriate sigmab value is nontrivial and depends on various aspects, such as measurement conditions and number of grains per aliquot (Galbraith and Roberts, 2012). Galbraith and Roberts (2012) emphasize that sigmab should be estimated individually for each sample and suggest using the central age model according to Galbraith et al. (1999) for that purpose. In our example case the function calc_CentralDose() reveals an overdispersion of 0.42 . Incorporating this value as sigmab improves the MAM performance in that the resulting minimum dose is less biased by the two lowermost values of the dose distribution (cf. graph of the KDE). The user may be reminded to carefully set sigmab according to the analysed data. The age model function returns model results as an "S4 object". Using the variable MAM, individual data objects were then adressed by get_RLum.Results(). The data object "results" was called to derive the minimum dose.

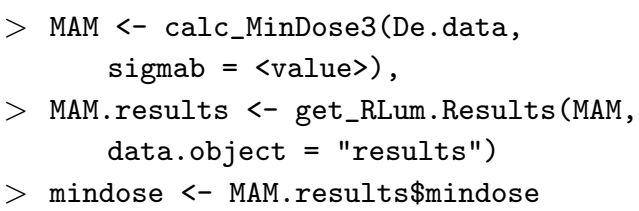

The finite mixture model (FMM, Galbraith and Green, 1990) is included in Figure 1 and template 3 (supplementary material). The FMM is not appropriate for the exemplary data set from the Pamir as multiple grain aliquots were used for measurements (e.g., Arnold and Roberts, 2009; Arnold et al., 2012), and the FMM is envisioned only for single grain data (Galbraith and Green, 1990). Nevertheless, the corresponding calc_FiniteMixture() is integrated in the template 3 (supplementary material) as an example of how to apply further age models. Apart from the sigmab, the FMM requires the number of components fitted to the dose distribution. The results of distribution unmixing are addressed by get_RLum.Results() and the data object "components". The $\mathbf{R}$ template 3 (supplementary material) shows how to routinely get the central values of individual components and determine the paleodose according to the FMM component with the highest proportion.

Age model results can be displayed, for example, using the plot_KDE() function. Adding lines by abline() for the minimum dose and FMM components identifies the parts of the dose distribution that MAM and FMM results are based on. Information on age model results was added to the legend by text () before saving the plot (for details see $\mathbf{R}$ template 3 supplementary material).

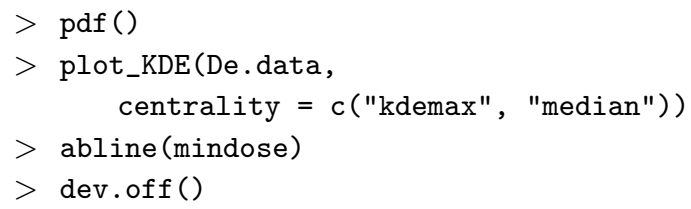

\section{Conclusions}

A fluvial sediment sample from the Pamir mountains is used to demonstrate the performance of the $\mathbf{R}$ package 'Luminescence' for OSL data analyses. We have described the data processing - including all steps from data evaluation after OSL measurements to statistical analyses using age models - in detail. We have illustrated how functions provided by the package can be combined via variables and $\mathbf{R}$ internal functions to take full advantage of the analytical and plotting possibilities of the $\mathbf{R}$ environment. The described workflow introduces one of many possible routines as an invitation to the wider geosciences community to use, adapt or combine the available functions and to contribute to the $\mathbf{R}$ package development. 


\section{Acknowledgments}

We are thankful to the $\mathbf{R}$ Coreteam for providing the $\mathbf{R}$ programming environment ( $\mathbf{R}$ Development Core Team, 2014) and the CRAN mirrors for open access to the package 'Luminescence' (http://cran.r-project.org/web /packages/Luminescence/index.html). We performed all analysis steps using the interface RStudio (http://www.rstudio.com/). Our ambition to develop and improve the $\mathbf{R}$ package 'Luminescence', is funded by the DFG network programm SCHM 3051/3-1. The work of the corresponding author was gratefully funded by the DFG in the frame of the TIPAGE bundle project (GI361/41) and benefited greatly from the supervision of Richard Gloaguen.

\section{References}

Arnold, L.J., Bailey, R.M., Tucker, G.E., 2007. Statistical treatment of fluvial dose distributions from southern Colorado arroyo deposits. Quaternary Geochronology 2, 162-167.

Arnold, L.J., Demuro, M., Ruiz, M.N., 2012. Empirical insights into multi-grain averaging effects from 'pseudo' single-grain OSL measurements. Radiation Measurements 47, 652-658.

Arnold, L.J., Roberts, R.G., 2009. Stochastic modelling of multigrain equivalent dose $\left(\mathrm{D}_{e}\right)$ distributions: Implications for OSL dating of sediment mixtures. Quaternary Geochronology 4, 204230 .

Bailey, R.M., Arnold, L.J., 2006. Statistical modelling of single grain quartz $\mathrm{D}_{e}$ distributions and an assessment of procedures for estimating burial dose. Quaternary Science Reviews 25, 2475-2502.

Bøtter-Jensen, L., Bulur, E., Duller, G.A.T., Murray, A.S., 2000. Advances in luminescence instrument systems. Radiation Measurements $32,523-528$.

Dietze, M., Kreutzer, S., Fuchs, M.C., Burow, C., Fischer, M., Schmidt, C., 2013. A practical guide to the $\mathrm{R}$ package Luminescence. Ancient TL 31, 11-18.

Duller, G.A.T., 2008. Single-grain optical dating of Quaternary sediments: Why aliquot size matters in luminescence dating. Boreas $37,589-612$.

Fuchs, M., Lang, A., 2001. OSL dating of coarse-grain fluvial quartz using single-aliquot protocols on sediments from NE-Peloponnese, Greece. Quaternary Science Reviews 20, 783-787.

Fuchs, M.C., Gloaguen, R., Pohl, E., 2013. Tectonic and climatic forcing on the Panj river system during the Quaternary. International Journal of Earth Sciences 102, 1985-2003.

Galbraith, R.F., Green, P.F., 1990. Estimating the component ages in a finite mixture. Nuclear Tracks and Radiation Measurements 17, 197-206.

Galbraith, R.F., Roberts, R.G., 2012. Statistical aspects of equivalent dose and error calculation and display in OSL dating: An overview and some recommendations. Quaternary Geochronology , 1-27.

Galbraith, R.F., Roberts, R.G., Laslett, G.M., Yoshida, H., Olley, J.M., 1999. Optical dating of single and multiple grains of quartz from Jinmium Rock Shelter, Northern Australia: Part I, Experimental design and statistical models. Archaeometry 41, 339-364.

Kreutzer, S., Schmidt, C., Fuchs, M.C., Dietze, M., Fischer, M., Fuchs, M., 2012. Introducing an R package for luminescence dating analysis. Ancient TL 30, 1-8.

Lepper, K., McKeever, S.W.S., 2002. An objective methodology for dose distribution analysis. Technical Report.

Murray, A.S., Olley, J.M., 2002. Precision and accuracy in the optically stimulated luminescence dating of sedimentary quartz: A status review. Geochronometria 21, 1-16.

Murray, A.S., Wintle, A.G., 2000. Luminescence dating of quartz using an improved single- aliquot regenerative-dose protocol. Radiation Measurements 32, 57-73.
Murray, A.S., Wintle, A.G., 2003. The single aliquot regenerative dose protocol: Potential for improvements in reliability. Radiation Measurements 37, 377-381.

Olley, J., Caitcheon, G., Murray, A., 1998. The distribution of apparent dose as determined by optical stimulated luminescence in small aliquots of fluvial quartz: Implications for dating young sediments. Quaternary Geochronology 17, 1033-1040.

R Core Team, 2014. R: A Language and Environment for Statistical Computing. R Foundation for Statistical Computing. Vienna, Austria. URL: http://www.R-project.org.

Rodnight, H., Duller, G.A.T., Wintle, A.G., Tooth, S., 2006. Assessing the reproducibility and accuracy of optical dating of fluvial deposits. Quaternary Geochronology 1, 109-120.

Thomsen, K.J., Bøtter-Jensen, L., Denby, P.M., Moska, P., Murray, A.S., 2006. Developments in luminescence measurement techniques. Radiation Measurements 41, 768-773. 